\title{
Sobre la idea de justicia en Marx $^{* 1}$
}

\author{
About Marx's justice idea
}

César Augusto Mora-Alonso

Universidad de Cartagena, Colombia

Recepción: 3 de marzo del 2017

Evaluación: 15 de junio del 2017

Aceptación: 4 de agosto del 2017

\footnotetext{
$*^{1}$. Este artículo ha sido posible por el Seminario de filosofía política contemporánea: teorías de la justicia, impartido por los profesores Juan José Botero Cadavid y Ángela Uribe Botero, durante el segundo semestre de 2014, para la Maestría en Filosofía, Convenio Universidad Nacional de Colombia-Universidad de Cartagena. A ellos, les expreso mis agradecimientos más sinceros por sus comentarios minuciosos, los cuales me obligaron a reflexionar sobre ciertos aspectos fundamentales.
}

Sobre la idea de justicia en Marx, 


\title{
Resumen
}

El artículo se concentra en indagar las diferentes declaraciones de Marx sobre la justicia, con la intención de determinar tres cuestiones: en primer lugar, saber si nuestro autor cuenta con una concepción de la justicia; en segundo lugar, establecer si la reflexión acerca de lo que es justo ocupa un lugar privilegiado en su obra; y, en tercer lugar, ver si a partir de ahí puede colegirse la existencia de una teoría marxiana de la justicia. De especial interés resulta la presencia de argumentos con implicaciones éticas y morales en estos asuntos.

Palabras clave: Marx; justicia; injusticia; distribución; dictados éticos.

\begin{abstract}
This article focuses on inquiring into Marx's different statements on justice, with the intention of determining three issues: first of all, to know whether the author has a conception of justice; secondly, to establish whether the reflection about what is fair occupies a privileged place in his work; and, thirdly, to see if from there the existence of a Marxian theory of justice can be concluded. Of particular interest is the presence of arguments with ethical and moral implications in these issues.
\end{abstract}

Key words: Marx; justice; injustice; distribution; ethical dictations. 


\section{INTRODUCCIÓN}

"La justicia en lo criminal es gratuita en todas partes; la justicia civil gira casi exclusivamente en torno a los pleitos sobre la propiedad y afecta, por tanto, casi únicamente a las clases poseedoras"

(Marx, 1977:20).

Lo que aquí me ha llevado a plantear el problema de la justicia en Marx ha sido, además de la propia lectura de sus textos, una afirmación que Robert van der Veen y Phillip van Parijs realizan en el célebre trabajo titulado "A Capitalist Road to Communism", a saber: "que para Marx las cuestiones de justicia y otras consideraciones éticas eran, si era que llegaban a ser relevantes, secundarias" (1986: 641)2.

Lo dicho por ellos no dejaba de resultarme inquietante, puesto que a Marx siempre se le ha relacionado con la denuncia de las iniquidades y con la promoción de un tipo de sociedad en la que imperaría el reino de la justicia, esto es, la comunista. Sin embargo, una revisión de la literatura especializada sobre el tema pone de manifiesto que la función que cumplen las ideas jurídicas y éticas en la obra de nuestro pensador ha estado sujeta a duras polémicas. Lo anterior se debe a que hay quienes sostienen que en dicha obra no existe una justificación de la justicia social, por más que muchas de las reivindicaciones en este sentido se encuentren amparadas en varios de sus pasajes.

De hecho, estudiosos de la talla de Allen. W. Wood (1972) y Norman Geras (1985) han orientado sus esfuerzos en demostrar que los ataques que dirige Marx contra el capitalismo no eran motivados por las injusticias que producía, ni que sus argumentos a favor del comunismo eran de índole ético. Por el contrario, Wood y Geras recuerdan que, para nuestro pensador, las razones de la superioridad del comunismo sobre el modo de producción capitalista pretendían estar basadas en el wissenschaftliche Einsicht, o sea, en el "conocimiento científico de la causa", en el "socialismo científico".

Tal vez, uno de los principales argumentos de corte "científico-objetivo" consistía en que el capitalismo representaba una fase acabada del desarrollo de las fuerzas productivas, que debía ser eliminada y sustituida por el modo de producción comunista. Esto, a razón de que la teoría materialista de los cambios históricos daba cuenta de que las relaciones de producción capitalistas ya interferían en el avance de las fuerzas productivas, pues limitaban

2 La traducción es mía. 
la opulencia e imponían la escasez, mediante el diseño de dispositivos tendientes a perpetuar la situación de pobreza innecesaria de la gran mayoría (Botero, 2005:190). En definitiva, el núcleo de la crítica al capitalismo reside en argumentos de pretendido orden científico que resaltan su obsolescencia y no las injusticias que parece ocasionar.

\section{LA JUSTICIA DEPENDE DEL MODO DE PRODUCCIÓN IMPERANTE}

Empero, aquí la pregunta obligada es qué entiende Marx por justicia. Una respuesta podría encontrarse en los Grundrisse (1985a) y, sobre todo, en la Crítica del Programa de Gotha (1977). En las obras referenciadas, el término "justicia" - como también el de "igualdad"- posee simplemente una acepción jurídica que da cuenta de la distribución económica en el modo de producción capitalista. Lo "justo", entonces, sería un patrón inherente a cada modo de producción, un corolario legal que se adapta a las condiciones materiales vigentes. Así que para Marx no tiene mucho sentido apelar a un concepto de justicia que tenga como base la "igualdad de derechos" o "el reparto equitativo", por cuanto son "patrañas ideológicas, jurídicas" y “tópicos en desuso" (1977:12) que suponen verdades que rebasan el plano de lo histórico.

En efecto, La ideología alemana (1994) y el Manifiesto comunista (1998) sostienen que conceptos como "justicia", "ley" o "libertad" son meras "formas de conciencia", que corresponden a los intereses de la clase dominante de una época, por lo que no poseen una existencia propia, sino que se encuentran sujetos a las relaciones de producción e intercambio imperantes. De acuerdo con Marx, tales conceptos darían la impresión de ser suprahistóricos — aun cuando sus significados cambien a lo largo de los siglos - por el hecho de que son "formas comunes de conciencia" que han estado presentes en la historia, la cual ha sido una historia de luchas entre clases opresoras y oprimidas. Dichas formas son el reflejo de la explotación y solo pueden desaparecer cuando se erradique el antagonismo de clases. "La revolución comunista es la ruptura más radical con las relaciones de propiedad tradicionales; no puede sorprender entonces que en su curso evolutivo se rompa de la manera más radical con las ideas tradicionales" (Marx y Engels, 1998:65).

Otra manera de decirlo: según Marx, una crítica radical al capitalismo, que invoque un concepto normativo de justicia distributiva, fracasa en su empeño, puesto que la distribución puede ser considerada como "justa" si responde plenamente al modo de producción imperante. Esto es lo que se plantea con suma perspicacia en la Crítica del Programa de Gotha: “¿Qué es reparto 
equitativo? ¿No afirman los burgueses que el reparto actual es equitativo? ¿Y no es este, en efecto, el único reparto 'equitativo' que cabe, sobre la base del modo actual de producción? ¿Acaso las relaciones económicas son reguladas por los conceptos jurídicos? ¿No surgen, por el contrario, las relaciones jurídicas de las relaciones económicas?" (1977:9)33.

Unas consideraciones similares se hallan en las "Glosas marginales al Tratado de Economía Política de Adolph Wagner", que aparecen como apéndice del primer volumen de El Capital:

Ahora bien, yo no presento nunca la ganancia del capitalista como una sustracción' o un 'robo' cometidos contra el obrero. Por el contrario, considero al capitalista como un funcionario indispensable del régimen capitalista de producción y demuestro prolijamente que no se limita a sustraer'o robar', sino que lo que hace es obtener la producción de plusvalía; es decir, que ayuda a crear ante todo aquello que ha de sustraer; y demuestro también por extenso que incluso en el cambio de mercancías se cambian solamente equivalentes y que el capitalista - siempre y cuando que pague al obrero el valor real de su fuerza de trabajo - tiene pleno derecho - dentro, naturalmente, del régimen de derecho que corresponde a este sistema de producción - a apropiarse de la plusvalía $(1974: 715)^{4}$.

Al respecto, también es célebre lo que se plantea en Salario, precio y ganancia:

Pedir una retribución igual, o simplemente una retribución equitativa, sobre la base del sistema del salariado, es lo mismo que pedir libertad sobre la base de un sistema esclavista. Lo que pudierais reputar justo o equitativo, no hace al caso. El problema está en saber qué es lo necesario e inevitable dentro de un sistema dado de producción (1976:44).

En síntesis, la justicia, para Marx, si es que puede hablarse de ella a partir de lo visto en estos pasajes, haría simplemente referencia al equilibrio entre las relaciones de producción y las fuerzas productivas en un modo de producción. Cualquier justificación de esto a partir de criterios éticos y morales sería pura ideología.

3 Todas las comillas simples y las palabras en cursivas que aparecen en las citas son de los autores mencionados.

4 De igual manera, en el tercer volumen de El Capital, se asegura que el "carácter justo" de las transacciones, que se dan entre los agentes involucrados en una relación comercial, encuentra su fundamento en el hecho de que tales transacciones son el resultado obvio de las relaciones de producción en un determinado modelo económico (1974:327). 


\section{LA DENUNCIA DE LAS INJUSTICIAS Y LOS DICTADOS ÉTICOS EN EL PENSAMIENTO MARXIANO}

Sin embargo, ¿por qué se vincula a Marx con la denuncia de las iniquidades y con la promoción de una sociedad justa? ¿Acaso están errados los movimientos y partidos políticos, de raigambre marxista, que tienen como lema básico el reclamo de justicia social? Una vez más, las respuestas a estos interrogantes podrían encontrarse en las obras de nuestro pensador, gracias a que, a lo largo de todas ellas, son palmarias las muestras de indignación ante los efectos del capitalismo. Y son precisamente estas muestras de indignación las que permiten considerar que Marx también percibe el problema con una mirada ética ${ }^{5}$.

Esta mirada, incluso, podría hallarse implícita en la tesis que sostiene que la justicia es el equilibrio entre las relaciones de producción y las fuerzas productivas en un modo de producción, puesto que, cuando las relaciones de producción empiezan a entorpecer el desarrollo de las fuerzas productivas, entonces, adviene el conflicto social, la lucha de clases. Ya para estos momentos, "las circunstancias que producen la demanda de justicia son (...) las circunstancias en las cuales dicha demanda no puede satisfacerse adecuadamente" (Bilbeny, 1984:81).

A juicio de Marx, el arma predilecta para esta exigencia de justicia es la crítica radical e implacable del orden establecido ${ }^{6}$. En la Introducción a la crítica de la Filosofía del Derecho de Hegel, asegura que dicha crítica tiene como pasión esencial la indignación y como trabajo central la denuncia (2014:48). Su deber, como histórico-filosófica que es, consiste en "desenmascarar la enajenación de sí mismo en sus formas profanas, después de que haya sido desenmascarada la figura santificada de la enajenación del hombre por sí mismo" (2014:44).

\footnotetext{
5 El profesor Botero señala acertadamente cómo en Marx hay una paradoja en este aspecto, dado que en su obra conspiran contra la existencia de una teoría de la justicia, su "ontología del ser genérico", su explicación materialista de los cambios históricos y su "socialismo científico". Lo interesante es que estos tres elementos "pueden verse como despliegues de tres concepciones teóricas determinantes, 'cargadas' éticamente: una concepción del ser humano y de su alienación; una teoría de los cambios históricos y de la explotación; y, finalmente, un proyecto emancipatorio, la perspectiva del comunismo" (2005:180).

6 Sin embargo, esta promoción de condiciones de justicia — que Marx parece impulsar mediante la denuncia y erradicación de las iniquidades - no lo convierte en un simple reformista social, ya que su objetivo no es ajustar al capitalismo para superar sus limitaciones; por el contrario, lo que busca es su demolición a través de una revolución proletaria.
} 
Además de tener que explicar las causas de la iniquidad, dicha crítica busca que los oprimidos sean conscientes del estado de postración en que se encuentran. Debe hacer que el hombre sea "el ser supremo para el hombre", debe concluir "en el imperativo categórico de acabar con todas las situaciones que hacen del hombre un ser envilecido, esclavizado, abandonado, despreciable. Nada mejor para describirlas que la exclamación de aquel francés ante el proyecto de un impuesto sobre los perros: ‘PPobres perros! ¡Os quieren tratar como a hombres!’” (2014:61).

En los Manuscritos de Economía y Filosofía (1985b), Marx explica con más detalle cómo se da esa explotación del hombre por el hombre. Lo que muestra es que las relaciones de producción del capitalismo originan y mantienen una división entre los pocos que poseen los medios de producción (los propietarios) y los muchos que solo poseen la fuerza de trabajo para subsistir (los trabajadores u obreros desposeídos). Estos últimos siempre llevan las de perder, pues quedan reducidos a la más barata de las mercancías y su penuria es inversamente proporcional a toda la riqueza que producen. Por eso, afirma que los trabajadores están enajenados no solo porque la riqueza que generan no les pertenece, sino también porque no tienen poder sobre su propia vida, ya que sus actividades laborales no son voluntarias sino forzadas. Al finalizar el "Primer Manuscrito", Marx señala que el trabajo enajenado es la causa tanto de la propiedad privada como del salario.

En "Sobre la cuestión judía" (2012), hay un duro cuestionamiento contra la propiedad privada y el dinero, pues ambos constituyen los fundamentos de la sociedad burguesa. Sin excepción, todo gira en torno a ellos: la política, el Derecho, la justicia y hasta los propios seres humanos. A juicio de Marx, lo que impera en esa sociedad es el afán desmedido de lucro y el egoísmo. Por esa razón, señala que la emancipación política no es suficiente, pues los derechos y el Estado burgués mantienen intacta la estructura de la enajenación.

Es aquí cuando cobra un papel vital el concepto de “emancipación humana". Un concepto, a todas luces, con una fuerte carga ética. Marx lo concibe de la siguiente manera:

Toda emancipación consiste en reabsorber el mundo humano, las situaciones y relaciones, en el hombre mismo. La emancipación política es la reducción del hombre por una parte a miembro de la sociedad burguesa, al individuo independiente y egoista, por la otra, al ciudadano, la persona moral. Solo cuando el hombre real, individual, reabsorba en sí mismo al abstracto ciudadano 
$\mathrm{y}$, como hombre individual, exista a nivel de especie en su vida empírica, en su trabajo individual, en sus relaciones individuales; solo cuando, habiendo reconocido y organizado sus fuerzas propias' como fuerzas sociales, ya no separe de sí la fuerza social en forma de fuerza política; solo entonces, se habrá cumplido la emancipación humana (2012:37).

Ahora bien, de acuerdo con nuestro pensador, el proletariado es quien tiene la responsabilidad de llevar a cabo esta emancipación, debido a que padece todo el rigor de la miseria, puesto que es una clase "con cadenas radicales (...) al que su sufrimiento universal confiere carácter universal; que no reclama un derecho especial, ya que no es una injusticia especial la que padece, sino la injusticia a secas (...)" (2014:72-73). Por ello, considera que la emancipación del proletariado se da cuando se libera de $-\mathrm{y}$ libera a su vez - las otras esferas de la sociedad, dado que encarna "la pérdida total del hombre, y por tanto, solo recuperándolo totalmente puede ganarse a sí misma" (2014:73).

En últimas, este propósito se expresa en lo que Marx llama Selbstbestimmung, autorrealización o autodeterminación. Consistente en el hecho de que cada persona tenga poder sobre su propia existencia, con la finalidad de que pueda desplegar todas sus capacidades de modo cabal y espontáneo. Lo que únicamente es posible cuando los proletarios logren que los antagonismos de clase y la división del trabajo sean eliminados. A partir de ahí, como reza el Manifiesto, surgirá una sociedad en la que el libre desarrollo de cada cual resulta ser el requisito para el libre desarrollo de todos (Marx y Engels, 1998:67).

Esta sociedad no es otra que la comunista. En ella, se dan todas las condiciones para que la producción sea regulada, de tal manera que a sus integrantes les sea posible desenvolverse en las actividades $u$ oficios de su predilección, y no queden compelidos al ejercicio de aquellos que no son de su interés. Esto permite:

Que yo pueda dedicarme hoy a esto y mañana a aquello, que pueda por la mañana cazar, por la tarde pescar y por la noche apacentar el ganado, y después de comer, si me place, dedicarme a criticar, sin necesidad de ser exclusivamente cazador, pescador, pastor o crítico, según los casos (Marx y Engels, 1994:46).

Con base en esta perspectiva, en el tercer volumen de El Capital (1974), se establece la distinción entre el "reino de la necesidad" y el "reino de 
la libertad". El primero se caracteriza por ser común a todas las formas de sociedad y a todos los modos de producción, incluso al comunista, puesto que los seres humanos siempre tendrán que saciar sus necesidades vitales; en otras palabras, se trata de mantener la supervivencia. Marx afirma que en este reino las necesidades experimentan crecimiento constante, pero que a la par se incrementan las fuerzas productivas que las satisfacen. De ahí que la libertad del "reino de la necesidad" resida en que los productores asociados puedan ajustar el funcionamiento de su sistema con el mundo natural. La idea es que controlen todos los mecanismos de su sistema productivo para que este no los domine como un poder ciego, además de ejecutarlo bajo condiciones dignas o que requieran poco esfuerzo.

Mas esta libertad que surge de la satisfacción de las necesidades - y que se ve expresada en la disminución drástica de la jornada laboral - constituye el requisito principal para el nacimiento del "verdadero reino de la libertad", que se caracteriza por encontrarse allende del ámbito de producción material y del trabajo marcado por la necesidad. De acuerdo con Marx, el fin supremo de este segundo reino es el desarrollo total de las potencialidades humanas, lo cual solo puede ser posible en una sociedad comunista.

Así pues, emancipación, autorrealización y libertad son los imperativos éticos que definen al pensamiento marxiano. En ellos tres, la humanidad encuentra su carácter genérico (Marx, 1985b). El artículo titulado "Los debates sobre la libertad de prensa y la publicación de los debates de la Dieta", aparecido en la Gaceta Renana, pone de relieve lo fundamental que resulta ser la libertad para nuestro pensador. De ella asegura lo siguiente:

La libertad es a tal punto la esencia del hombre, que hasta sus adversarios la realizan cuando luchan contra su realidad; tratan de apropiarse como la joya más valiosa lo que rechazan como joya de la naturaleza humana. Nadie combate la libertad; combate, a lo sumo, la libertad de los otros. Por tanto, todas las libertades han existido siempre, primero como privilegio particular de unos y luego como el derecho general de todos (1982:194).

No obstante, la concepción marxiana de la libertad difiere de la ostentada por la tradición liberal, dado que esta última - con su lema de hacer y deshacer, siempre y cuando no se perjudique al otro, y cuya aplicación práctica se resume en el derecho de la propiedad privada - termina por convertir a las personas en mónadas aisladas y replegadas. En una sociedad en la que prima 
el egoísmo y la desunión, lo que puede hallar un individuo en otro no es la realización, sino más bien la restricción de su libertad (Marx, 2012:32) ${ }^{7}$.

De ahí que el ejercicio de esta solo pueda ser posible en lo que Marx denomina Gemeinschaft, comunidad, por la razón de que en la susodicha el ser humano despliega todo su ser social, su auténtica naturaleza cooperativa (Marx, 1985b). Lo anterior hace posible que la expresión individual de una vida tenga en cuenta la de los demás, ya que en las relaciones sociales debe primar, ante todo, la reciprocidad y no el provecho particular a expensas del perjuicio general. Sin embargo, esto no representa un óbice para el desarrollo de los proyectos personales de vida, puesto que el comunismo se caracteriza por ser una asociación de hombres y mujeres libres en actividad libre, en la que el libre desarrollo de cada cual representa una condición necesaria para el libre desarrollo de todos (Marx y Engels, 1998:67).

\section{LOS DOS PRINCIPIOS DE JUSTICIA EN LA CRÍTICA DEL PROGRAMA DE GOTHA}

Pero, como se sabe, para poder llegar a este "auténtico reino de la libertad", hay que pasar por el socialismo. De acuerdo con el Manifiesto, en esta primera etapa revolucionaria, lo fundamental es que el proletariado tome el poder político, con el objetivo de apoderarse gradualmente de todos los bienes de la clase burguesa. Lo que se busca con ello es la centralización de los medios de producción y el aumento acelerado del conjunto de las fuerzas productivas. Aunque, en la Crítica del Programa de Gotha, se aclara que en este periodo de transición resulta inevitable la presencia de ciertos defectos del capitalismo, uno de los cuales es el intercambio de mercancías equivalentes.

Aun cuando la manera como se da ese intercambio en el régimen socialista difiere en aspectos cruciales de su similar capitalista — dado que la propiedad se convierte en colectiva, al margen de los medios individuales de

\footnotetext{
Es cierto que Marx miraba con recelo las libertades burguesas debido a su estrecha conexión con el egoísmo y el beneficio individual. No obstante, como lo anota muy bien G. Restrepo (1999), también es cierto que Marx resalta el valor y el significado de estas libertades, si se llegan a comparar con las limitaciones a la libertad en la época medieval. Nuestro pensador es consciente del papel que desempeñan en las luchas del proletariado por mejorar sus condiciones laborales y, sobre todo, por el establecimiento de las condiciones propicias para el advenimiento de la "verdadera libertad". Una vez más, G. Restrepo observa con perspicacia que el Manifiesto comunista se va lanza en ristre contra los derechos económicos (libertades de empresa, contratación y apropiación), pero que no sucede lo mismo con los derechos individuales y políticos (libertades de pensamiento, expresión y asociación).
} 
consumo, y en estas condiciones ya nadie puede dar sino su trabajo-, lo cierto es que en la distribución continúa imperando el mismo principio de las transacciones, por cuanto "se cambia una cantidad de trabajo, bajo una forma, por otra cantidad igual de trabajo, bajo otra forma distinta" (Marx, 1977:11). Y, lo que parece ser un principio de reparto equitativo, termina por convertirse en una distribución desigual. En ello reside el defecto principal de esta etapa de transición. Al respecto, afirma Marx:

Pero unos individuos son superiores, física e intelectualmente a otros y rinden, pues, en el mismo tiempo, más trabajo, o pueden trabajar más tiempo; y el trabajo, para servir de medida, tiene que determinarse en cuanto a duración o intensidad; de otro modo, deja de ser una medida. Este derecho igual es un derecho desigual para trabajo desigual. No reconoce ninguna distinción de clase, porque aquí cada individuo no es más que un trabajador como los demás; pero reconoce, tácitamente, como otros tantos privilegios naturales, las desiguales aptitudes de los individuos y, por consiguiente, la desigual capacidad de rendimiento. En el fondo es, por tanto, como todo derecho, el derecho de la desigualdad (1977:11-12).

La situación cambia en la etapa superior del comunismo, pues el desarrollo de la productividad y de las condiciones de trabajo hace posible un grado de abundancia, en el que ya no es necesario igualar de manera artificial a los individuos a través de un conjunto de derechos; a razón de que ya se han superado los antagonismos de clase, de manera que cada quien puede realizar libremente su individualidad y contribuir, al mismo tiempo, al fomento de la de sus semejantes. Marx describe esta etapa suprema de la siguiente manera:

En una fase superior de la sociedad comunista, cuando haya desaparecido la subordinación esclavizadora de los individuos a la división del trabajo, y con ella, el contraste entre el trabajo intelectual y el trabajo manual; cuando el trabajo no sea solamente un medio de vida, sino la primera necesidad vital; cuando, con el desarrollo de los individuos en todos sus aspectos, crezcan también las fuerzas productivas y corran a chorro lleno los manantiales de riqueza colectiva, solo entonces podrá rebasarse totalmente el estrecho horizonte del derecho burgués y la sociedad podrá escribir en sus banderas: ¡De cada cual según sus capacidades; a cada cual según sus necesidades! (1977:12).

Sin embargo, no deja de llamar la atención el hecho de que estas consideraciones sobre el socialismo y el comunismo se realicen, en la Crítica del Programa de Gotha, en el contexto de las fuertes objeciones que dirige Marx 
contra el ideal igualitario de los obreros alemanes, puesto que el objetivo fundamental no debe ser el simple reclamo por una igualdad de derechos, sino el alcance de una sociedad que haya rebasado las limitaciones de la sociedad burguesa. Por eso, el verdadero carácter revolucionario del Partido Obrero Socialista de Alemania debe consistir en la adopción del "socialismo científico" y el abandono o rechazo de las posiciones del "socialismo vulgar", que pierden de vista la perspectiva del comunismo (Botero, 2005:188).

De ahí que Marx califique como un error sostener que el "socialismo científico" sea una doctrina que gire única y exclusivamente en torno a la distribución o que le asigne a esta un papel preponderante; ya que dicha distribución, como se ha indicado, no es independiente del modo de producción, sino que es su reflejo fiel. A pesar de ello, como también se ha visto, nuestro pensador no soslaya la función que cumple la distribución en el equilibrio entre las relaciones de producción y las fuerzas productivas en un modo de producción. De hecho, en La ideología alemana, reconoce que con la división del trabajo - y, de manera especial, con la separación entre el trabajo físico y el intelectual - surge la distribución "y, concretamente, la distribución desigual, tanto cuantitativa como cualitativamente, del trabajo y de sus productos (...)" (1994:45).

Esto último abre la puerta para hablar en el pensamiento marxiano de "justicia" e "injusticia" en la distribución. A esta tesis llega J. Elster en Making Sense of Marx. En su opinión, los principios para una distribución justa se hallarían implícitos en la distinción establecida entre las dos etapas del comunismo en la Crítica del Programa de Gotha: curiosamente, en el pasaje en que Marx esgrime argumentos demoledores contra las teorías de la justicia por operar a partir de principios generales y abstractos. De acuerdo con Elster, lo que en realidad nuestro pensador no supo fue que terminó por invocar principios de justicia similares a los que pretendía rechazar. En el socialismo, o etapa de transición en la que se impone la dictadura del proletariado, regiría el de la distribución según la contribución aportada; entre tanto, en la etapa propiamente comunista, lo haría el principio de la distribución según las necesidades (Elster, 1999:222).

El principio de distribución según la contribución aportada se caracteriza por remunerar a los trabajadores en función del tiempo de trabajo que proporcionan, es decir, cada trabajador percibe un sueldo por la jornada laboral cumplida. Aquí "la sociedad le entrega un bono consignado que ha rendido 
tal o cual cantidad de trabajo (...), y con este bono saca de los depósitos sociales de medios de consumo la parte equivalente a la cantidad de trabajo que rindió" (Marx, 1977:11). Este principio aspira a que los productores asociados sean remunerados equitativamente, por eso la distribución se efectúa en términos de una igualdad de derechos. El problema es que esta termina por desembocar en una distribución desigual, pues si la igualdad consiste en medirlos a todos por la pauta del trabajo, habrá que reconocer, entonces, que unos individuos son más productivos que otros.

Elster afirma que este principio marxiano de la contribución aportada puede ser abordado desde dos enfoques, ya que, de un lado, vale como criterio de justicia para condenar la explotación capitalista como injusta; pero, de otro lado, se queda corto ante el patrón expresado por el principio de distribución según las necesidades, que caracteriza a la etapa superior del comunismo (1999:229-230).

Precisamente, este último principio se distingue del primero por no ser de corte igualitarista. La razón estriba en que los derechos son trascendidos en la fase suprema del comunismo, dado que se ha establecido un régimen de abundancia que posibilita la desaparición de intereses contrapuestos entre los individuos. En ese sentido, no resulta indispensable un sistema de derechos que se encargue de conciliar los intereses en conflicto; estos brillarán por su ausencia al haberse vencido los obstáculos — sobre todo, de tipo materialque entorpecen la autorrealización humana. Y, aunque es verdad que el comunismo es una sociedad sin clases, eso no implica que todos los individuos sean considerados como iguales; por el contrario, el principio de distribución según las necesidades lo que busca es el desarrollo integral de los distintos proyectos personales de vida.

En resumen, para Elster, Marx elaboró una teoría de la justicia en términos jerárquicos, en la que el principio de distribución según la contribución aportada ofrece el segundo mejor criterio, cuando el principio de distribución según las necesidades no reúne todavía las condiciones históricas para su puesta en práctica. En este orden de ideas, el capitalismo es injusto por cuanto no responde a ninguno de estos dos principios. El socialismo también es injusto, pero, claro está, menos que su similar capitalista, ya que únicamente quebranta el principio de distribución según las necesidades (1999:230). Así pues, el comunismo es más justo que el socialismo y, a su vez, este lo es más que el capitalismo. 


\section{Conclusión}

Dos han sido los motivos que me han llevado a la redacción de este artículo: esclarecer la idea de justicia en Marx y determinar si esta ocupa un lugar privilegiado en su obra. Desde el principio, tales cuestiones revelaron su complejidad, pues, por un lado, siempre se ha vinculado a este pensador con la denuncia de las iniquidades y con la promoción de una sociedad más justa; pero, por otro lado, aparece el hecho de que sus críticas al modo de producción capitalista y sus argumentos sobre la superioridad del comunismo no pretenden estar basados en criterios de justicia ni en juicios de carácter normativo.

De hecho, se pudo apreciar la actitud despectiva que en ocasiones Marx parece tener frente a todo tipo de consideraciones éticas, puesto que llega a afirmar que ideales como los de libertad, derecho y moralidad son simples formas de conciencia que revelan los intereses de las clases hegemónicas, razón por la cual, para él, no poseen una existencia suprahistórica, sino que se hallan sometidos a las relaciones de producción e intercambio históricamente condicionadas. En la misma situación se encuentra a la justicia, ya que esta solo expresaría el equilibrio entre las relaciones de producción y las fuerzas productivas en cualquier sistema económico-político; es decir, la justicia, en su opinión, simplemente sería un corolario legal que se adapta a las condiciones materiales vigentes, cuya única finalidad es dar cuenta de la distribución de recursos en un modo de producción. En suma, la justicia depende del modo de producción, con lo que quedaría descartado un concepto normativo sobre ella o su justificación a partir de criterios éticos y morales.

Sin embargo, también se vio que en la obra de Marx abundan las muestras de indignación frente a los efectos adversos que genera el capitalismo: concretamente, la explotación y miseria a las que se ven sometidas las grandes mayorías. Aquí tanto la denuncia de las iniquidades como la exigencia de mejores condiciones de vida pasa por la crítica tajante del statu quo. La finalidad suprema de dicha crítica consiste, entonces, en erradicar por completo las situaciones de alienación y opresión. Es en este punto en el que aparece toda la dimensión ética del pensamiento marxiano, la cual puede recogerse en tres imperativos: emancipación humana, autorrealización y libertad; aunado a ellos, la auténtica vida comunitaria. Solo de esta manera, para nuestro 
pensador, los seres humanos pueden alcanzar su naturaleza genérica y cooperativa $^{8}$. La sociedad comunista sería la encarnación de estos valores.

Lo anterior puso de relieve que en la obra de Marx es posible encontrar, pese a todas sus prevenciones y advertencias, argumentos con implicaciones éticas y morales que resaltan la superioridad del comunismo sobre otros modos de producción; en especial, sobre el capitalismo. Lo que permite el hallazgo implícito de una valoración similar en términos de justicia e injusticia. Precisamente, a esto ha apuntado la tesis de Elster, quien sostiene que Marx elaboró una teoría jerarquizada de la justicia compuesta por dos criterios -el de la distribución, según la contribución aportada y el de la distribución, según las necesidades - que permite el juzgamiento de capitalismo, socialismo y comunismo.

Ahora bien, en función de todo lo expuesto, se podría preguntar si las reflexiones sobre la justicia ocupan un lugar privilegiado en el pensamiento de Marx. A mi juicio, la respuesta tendría un carácter afirmativo por partida doble. En primer lugar, porque en su escrutinio del modo de producción capitalista resulta imprescindible la referencia al vínculo que en él mantienen las fuerzas productivas y las relaciones de producción. Como ya se dijo, el equilibrio resultante entre esos dos aspectos inseparables al modo de producción es lo que Marx llega a concebir como justicia. A este respecto, su crítica de la economía política explica detalladamente que la forma de distribución —con todas las consecuencias que acarrea - es la expresión natural de cualquier sistema productivo, y que este último determina por completo el régimen social. En este orden de ideas, la obra de Marx es un diagnóstico de la sociedad capitalista y sus instituciones.

8 En relación con esto, conviene hacer la siguiente observación: Marx identifica "esencia humana" y "naturaleza humana" en los Manuscritos de Economía y Filosofía. Sin embargo, en las Tesis sobre Feuerbach, nuestro autor muestra sus reservas con la primera expresión por su carácter genérico y abstracto. De ahí que señale que la esencia humana es en realidad el conjunto de las relaciones sociales, y que el enfoque del nuevo materialismo deba ser la sociedad humana o la humanidad socializada (Bendaña, 1981:80-83). El punto es que en el primer volumen de El Capital emplea de manera positiva la expresión "naturaleza humana", tanto así que habla de "naturaleza humana en general" y "naturaleza humana históricamente condicionada". Lo anterior da pie para que E. Fromm (1970) advierta que, en lo tocante a la concepción de la esencia humana, exista una continuidad entre los planteamientos de los Manuscritos y El Capital. De acuerdo con el filósofo francfortiano, la expresión "naturaleza humana" de El Capital conserva la idea de "esencia humana" de los Manuscritos. La cuestión es que esta no se emplea explícitamente para efectos de un tratamiento histórico y concreto de las temáticas de economía política (1970:36-37). A. Fazio (2013) destaca la importancia de ambas nociones en la obra de Marx, puesto que reflejan la continuidad de su pensamiento a través de los textos de juventud y madurez.

Sobre la idea de justicia en Marx,

César Augusto Mora-Alonso - p. 45-63 
En segundo lugar, porque cuando las relaciones de producción comienzan a obstaculizar el avance de las fuerzas productivas hay un recrudecimiento del conflicto social, de la lucha de clases. En este punto, los escenarios que provocan la exigencia de justicia son aquellos en los cuales dicha exigencia no logra satisfacerse. Por eso, lo que se busca es la subversión de los factores que originan y perpetúan las iniquidades. La máxima aspiración de la revolución proletaria es la instauración del comunismo. Un sistema que pretende superar las limitaciones del capitalismo, dado que acaba con la explotación, el trabajo enajenado, la propiedad privada y el régimen de salarios, fundamentos sobre los que se erige la sociedad del capital. Tanto la obra de Marx como su praxis política estuvieron consagradas a la consecución de un sistema que garantizara las mejores condiciones de vida para todos.

Pero, ¿sería lícito, a partir de estas consideraciones, hablar de una teoría marxiana de la justicia? En mi opinión, obtener una respuesta contundente no sería una tarea fácil. Por un lado, habría elementos de juicio que inclinarían la balanza hacia el sí. A. Sen (2009), por ejemplo, plantea que Marx ofrece una base intelectual rigurosa con el fin de transitar de un sentimiento general de injusticia a diagnósticos particulares razonados de la injusticia, y de estos a los análisis de los modos de propugnar la justicia. Además, de acuerdo con Elster, nuestro pensador contaría con una teoría jerarquizada de la justicia, compuesta por dos principios, que regirían la distribución de recursos en el socialismo y el comunismo.

Por otro lado, sin embargo, habría argumentos de peso para decidirse por el no, pues el problema de la distribución justa ya no se presentaría en la etapa superior del comunismo. A juicio del profesor Botero, lo anterior se sustenta en la ausencia de lo que Hume y Rawls denominan circunstancias de justicia, a saber: escasez relativa e intereses contrapuestos. No existiría la primera porque el despliegue de las fuerzas productivas ha facilitado el establecimiento de un régimen de abundancia. Y no existiría la segunda porque los seres humanos han dejado de ser egoístas y ya no se requiere de la presencia de una organización estatal encargada de conciliar los intereses en conflicto (2005:183). Empero, el argumento más poderoso estaría en las declaraciones del propio Marx, quien — pese al lugar destacado que ocupó la reflexión sobre la justicia en su obra - siempre la consideró como algo accesorio y circunstancial al modo de producción.

Pienso, al igual que Elster, que la razón por la cual resulta complicado establecer la existencia de una teoría de la justicia en Marx radica en que hay 
una ambigüedad fundamental en su pensamiento. Dicha ambigüedad impide liquidar, de una vez por todas, el problema, ya que numerosos pasajes de su obra revelan una actitud hostil a la hora de tratar asuntos relacionados con la ética, los derechos y la justicia, mientras que otros dejan ver una actitud radicalmente distinta. Da la impresión de que, en el fondo, Marx contara con un rasero relativo y otro transhistórico para referirse a estos ideales.

En este sentido, me gustaría concluir con la cita de dos textos que ponen de relieve esta disyuntiva. El primero, tomado del Manifiesto comunista, reza del siguiente modo:

Pero no disputéis con nosotros midiendo la abolición de la propiedad burguesa con el patrón de medida de vuestras ideas de libertad, instrucción, justicia, etcétera. Vuestras propias ideas son producto de las relaciones burguesas de producción y propiedad, así como vuestra justicia es solo la voluntad de vuestra clase elevada a la categoría de ley, una voluntad cuyo contenido se halla dado en las condiciones materiales de vida de vuestra clase (Marx y Engels, 1998:61).

El segundo, que hace parte de los Estatutos generales de la Asociación Internacional de los Trabajadores, afirma:

Por todas estas razones ha sido fundada la Asociación Internacional de los Trabajadores. $Y$ declara: que todas las sociedades y todos los individuos que se adhieran a ella reconocerán la verdad, la justicia y la moral como base de sus relaciones recíprocas y de su conducta hacia todos los hombres sin distinción de color, de creencias o de nacionalidad. No más deberes sin derechos, no más derechos sin deberes (Marx, 1980:7).

La cuestión es que, al parecer, Marx nunca mostró interés en desarrollar una ética o filosofía moral que respaldara esta visión supuestamente transhistórica de la justicia. Entonces, Robert van der Veen y Phillip van Parijs tendrían la razón'.

\footnotetext{
9 Aunque eso no quiere decir que en la obra de Marx no se encuentre una perspectiva ética de base. La prueba, como vimos, se halla en los imperativos que se reflejan en sus denuncias sobre las condiciones de opresión y en las aspiraciones para la construcción de una sociedad mejor. Aquí residen los pilares sobre los cuales el pensamiento marxista ha construido y sigue construyendo su concepción del bienestar y la justicia.
} 


\section{REFERENCIAS}

Bendaña, C. (1981). Karl Marx: Tesis sobre Feuerbach. Bogotá: Félix Burgos Editor.

Bilbeny, N. (1984). ¿Hay una moral marxista? Enrahonar. Quaderns de Filosofia, (9), pp. 79-84. Barcelona: Universidad Autónoma de Barcelona.

Botero, J. (2005). Rawls, Marx y la justicia social. J. Botero (Ed.), Con Rawls y contra Rawls: una aproximación a la filosofía política contemporánea (pp. 179-192). Bogotá: Universidad Nacional de Colombia.

Elster, J. (1999). Making Sense of Marx. Cambridge: Cambridge University Press.

Fazio, A. (2013). De los Manuscritos de 1844 a El Capital: notas sobre ética y ontología en el pensamiento de Carlos Marx. $\Delta \alpha i \mu \omega v,(58)$, pp. 95-108. Murcia: Universidad de Murcia.

Fromm, E. (1970). Marx y su concepto del hombre. México: FCE.

Geras, N. (1985). The Controversy about Marx and Justice. New left review, (150), pp. 47-85. Londres: NLR.

Marx, K. (1974). El Capital. (Vols. I y III). (Trad. W. Roces). México: FCE.

-----. (1976). Salario, precio y ganancia. (Trad. Ediciones en lenguas extranjeras). Beijing: Ediciones en lenguas extranjeras.

------. (1977). Crítica del Programa de Gotha. (Trad. Editorial Progreso). Moscú: Editorial Progreso.

------. (1980). Estatutos generales de la Asociación Internacional de los Trabajadores. (Trad. Editorial Progreso). Moscú: Editorial Progreso.

------. (1982). Los debates sobre la libertad de prensa y la publicación de los debates de la Dieta. W. Roces (Ed.), Marx. Escritos de juventud (Trad. W. Roces) (pp. 173-219). México: FCE.

-----. (1985a). Grundrisse. (Trad. W. Roces). México: FCE.

------. (1985b). Manuscritos de Economía y Filosofía. (Trad. F. Rubio Llorente). Madrid: Alianza. 
-----. (1994). La ideología alemana. (Trad. W. Roces). Valencia: Universitat de València.

------ y Engels, F. (1998). Manifiesto comunista. (Trads. E. Grau Biosca y L. Mames). Barcelona: Crítica.

------. (2012). Sobre la cuestión judía. F. Groni (Ed.). Páginas malditas. Sobre La Cuestión Judia y otros textos (Trad. F. Groni) (pp. 13-46). Buenos Aires: Libros de Anarres.

------. (2014). Introducción a la crítica de la Filosofía del Derecho de Hegel. (Trad. J.M. Ripalda). Valencia: Pre-textos.

Restrepo, G. (1999). Ética y libertad en Marx. J. Caycedo y J. Estrada (Comps.), Marx Vive (pp. 139-153). Bogotá: Universidad Nacional de Colombia.

Sen, A. (2009). The Idea of Justice. Cambridge: Harvard University Press.

van der Veen, R., y van Parijs, Ph. (1986). A Capitalist Road to Communism. Theory and Society, (15:5), pp. 635-655. Dordrecht: Martinus Nijhoff Publishers.

Wood, A. W. (1972). The Marxian Critique of Justice. Philosophy and Public Affairs, (1:3), pp. 244-282. Princeton: Princeton University Press. 